\section{Delayed discharge: how are services and patients being affected?}

\begin{abstract}
As NHS hospitals in England contend with covid and winter illnesses, many are trying to free up capacity by clearing beds of patients who are fit for discharge. Matthew Limb looks at the challenges they face
\end{abstract}

Matthew Limb

\section{How many patients medically fit for discharge are currently occupying beds?}

The latest figures, from 9 January 2022, show that NHS hospitals in England had 17303 patients who no longer met the "criteria to reside." Of these, 4907 were discharged, leaving 12396 in hospital.

\section{What discharge targets have been set?}

In December NHS hospital trusts and other NHS bodies were told to discharge at least half of patients who were medically fit to leave, in preparation for a surge in cases of covid caused by the omicron variant and to "release the maximum number of beds." Patients who don't need an NHS bed must be discharged safely "as soon as practically possible," said the guidance from NHS England and NHS Improvement. ${ }^{2}$

\section{What is causing the delays?}

Data on causes are no longer collected centrally. As recently as February 2020 the NHS was responsible for $60 \%$ of discharge delays. ${ }^{3}$ But experts have said a key cause of the current delays is the pressured situation in social care and, in particular, home care. "In social care, services are struggling to meet the needs of people already in receipt of social care, and they simply don't have capacity to take on new clients who are being discharged from hospital," said Natasha Curry, deputy director of policy at the health think tank the Nuffield Trust.

\section{How are hospitals doing?}

Hospital trusts accept that delayed discharge is bad for patient flow through the system and for outcomes among patients. They're trying to focus on those patients who can be discharged home without the need for social care support. They are also working with local authorities and other partners, including hospices and care homes, to release the maximum number of beds. But the Health Service Journal has reported that many trusts are struggling to meet the 50\% target. ${ }^{4}$ Miriam Deakin, director of policy and strategy at NHS Providers, said, "This target is a significant ask of providers at a time of increased operational pressure."

Deakin added that success was "contingent on having enough staff" to facilitate discharge and to support patients once they are discharged.

\section{What action is being taken?}

In the week beginning 10 January hospitals across Kent, Sussex, and Surrey were asked to discharge hundreds of patients who were well enough to leave by the end of the week. This included University Hospitals Sussex NHS Foundation Trust, which said it was working with community partners to discharge 232 patients in its hospitals who were medically ready for discharge. "Once patients are medically ready to leave hospital, we need their families, carers, or social care settings to support them as much as necessary to be able to go home safely," said Maggie Davies, chief nurse.

Other areas are using hotels, including NHS Devon Clinical Commissioning Group and its local partners, which are making use of 32 beds in local hotels, with specialist care and support for people who would otherwise be stuck in hospital waiting for support at home. The CCG said, "Feedback from patients has been excellent, and since March 2020 this has saved several thousand hospital bed days. This has freed beds for people who needed inpatient care in a hospital setting."

\section{What instructions have community providers received?}

Community providers have been asked by NHS England and NHS Improvement to deprioritise "low priority cases" across several services so that they can redeploy staff to support the vaccination programme and hospital discharges. But Deakin said this measure "may raise concerns about exacerbating community care backlogs, widening inequalities, and jeopardising the delivery of post-discharge support in the community, particularly if the number of patients discharged from hospital settings increases in line with the targets set by NHSE/I."

\section{What do doctors' leaders say?}

Katherine Henderson, president of the Royal College of Emergency Medicine, said freeing up beds will help to "prevent exit block, dangerous crowding, and corridor care" but added, "It is often the case that long stay patients are the most vulnerable who need support from social care services in their return to the community. The ongoing social care crisis is preventing these vulnerable patients from safely returning home or to their community when they have completed their treatment."

Meanwhile, the BMA has warned that the "rapid discharge" of patients into community settings could have a destabilising effect on already stretched general practices. Farah Jameel, chair of the BMA's General Practitioners Committee, has warned NHS bosses, "As it currently stands, there remains insufficient capacity, and wholly inadequate support across both general practice and community care 
teams, to meet the ongoing care and treatment needs of patients." 5

\section{What's the view of social care?}

Nadra Ahmed, chair of the National Care Association, whose 700 members include small to medium size residential and nursing homes and home care providers, said that pressure on the sector was as bad as it was in the NHS, if not worse. "Nationally, in some local authority areas, up to two thirds of care services are not open to admissions, because of staff shortages and covid outbreaks, and we have home care agencies who are handing back contracts because they can't service them," she said.

\section{Are there risks to patients?}

Curry said that although people needed to meet certain criteria to be discharged, effective discharge often depended on them having adequate support once they left hospital. "If social care services are struggling to meet demand, one possible consequence is that unpaid carers have to fill the gap and support people with complex needs, putting more pressure on them," she said. "If support is not adequate or it breaks down, the risk of readmission could be heightened." One concern it that unpaid carers are under immense pressure and that breakdown in caring arrangements are fuelling demand for formal social care.

"Another risk is that people are discharged not to an ideal setting, so they might go to a care home, where ideally if the right support was put in place at home they could go home," Curry added.

\section{What measures might improve matters?}

David Fothergill, who chairs the Local Government Association's Community Wellbeing Board, said a bigger proportion of the new health and social care levy "should go directly towards social care upfront" to help deal with immediate pressures.

In the longer term, Rory Deighton, senior programme lead for acute care at the NHS Confederation, said that boosting the social care workforce was crucial. "While the NHS will continue to do everything it can to prioritise patients with the greatest clinical need and discharge patients ready to leave hospital as safely and quickly as possible, without a long term, properly funded strategy to increase the social care workforce healthcare leaders are worried this situation will worsen," he said.

1 NHS England. Urgent and emergency care daily situation reports 2021-22. https://www.england.nhs.uk/statistics/statistical-work-areas/uec-sitrep/urgent-and-emergency-care-daily-situationreports-2021-22

2 NHS England. Preparing the NHS for the potential impact of the Omicron variant and other winter pressures. Dec 2021. https://www.england.nhs.uk/coronavirus/publication/preparing-the-nhsfor-the-potential-impact-of-the-omicron-variant-and-other-winter-pressures.

3 Nuffield Trust. Delayed transfers of care. Feb 2021. https://www.nuffieldtrust.org.uk/resource/delayed-transfers-of-care.

4 Discombe M. Trusts fall far short of NHSE discharge target. HSJ. 7 Jan 2022. https://www.hsj.co.uk/quality-and-performance/trusts-fall-far-short-of-nhse-discharge-target/7031659.article.

5 Jameel F. Letter from BMA to Stephen Powis, NHS national medical director. Dec 2021 https://i.emlfiles4.com/cmpdoc/3/7/7/5/2/files/867893_bma-gpc-to-prof-steve-powis-24december21.pdf. 